\title{
Second harmonic imaging using synthetic aperture sequential beamforming
}

\author{
Du, Yigang; Rasmussen, Joachim; Jensen, Henrik; Jensen, Jørgen Arendt
}

Published in:

Proceedings of the 2011 IEEE International Ultrasonics Symposium

Link to article, DOI:

10.1109/ULTSYM.2011.0561

Publication date:

2011

Document Version

Early version, also known as pre-print

Link back to DTU Orbit

Citation (APA):

Du, Y., Rasmussen, J., Jensen, H., \& Jensen, J. A. (2011). Second harmonic imaging using synthetic aperture sequential beamforming. In Proceedings of the 2011 IEEE International Ultrasonics Symposium (pp. 22612264). IEEE. https://doi.org/10.1109/ULTSYM.2011.0561

\section{General rights}

Copyright and moral rights for the publications made accessible in the public portal are retained by the authors and/or other copyright owners and it is a condition of accessing publications that users recognise and abide by the legal requirements associated with these rights.

- Users may download and print one copy of any publication from the public portal for the purpose of private study or research.

- You may not further distribute the material or use it for any profit-making activity or commercial gain

- You may freely distribute the URL identifying the publication in the public portal 
Paper presented at the IEEE International Ultrasonics Symposium, Orlando Florida, 2011:

\section{Second harmonic imaging using synthetic aperture sequential beamforming}

Yigang Du ${ }^{1,2}$, Joachim Rasmussen ${ }^{1}$, Henrik Jensen ${ }^{2}$ and Jørgen Arendt Jensen ${ }^{1}$

${ }^{1}$ Center for Fast Ultrasound Imaging,

Biomedical Engineering group, Department of Electrical Engineering, Bldg. 349,

Technical University of Denmark, DK-2800 Kgs. Lyngby, Denmark

${ }^{2}$ BK Medical Aps, Mileparken 34, DK-2730 Herlev, Denmark,

To be published in Proceedings of IEEE International Ultrasonics Symposium, Orlando Florida, 2011. 


\title{
Second harmonic imaging using synthetic aperture sequential beamforming
}

\author{
Yigang $\mathrm{Du}^{1,2}$, Joachim Rasmussen ${ }^{1}$, Henrik Jensen ${ }^{2}$ and Jørgen Arendt Jensen ${ }^{1}$ \\ 1) Center for Fast Ultrasound Imaging, Department of Electrical Engineering, Bldg. 349, \\ Technical University of Denmark, DK-2800 Kgs. Lyngby, Denmark. \\ 2) BK Medical Aps, Mileparken 34, DK-2730 Herlev, Denmark.
}

\begin{abstract}
The paper investigates Second Harmonic Imaging (SHI) using Synthetic Aperture Sequential Beamforming (SASB). The investigation is made by an experimental Synthetic Aperture Real-time Ultrasound System (SARUS). A linear array transducer is used to scan 4 wires at the image depths of $\{22.5$, 47.5, 72.5, 97.5 $\mathrm{mm}$, respectively. Three different experiments are made using three different transmit foci at $10 \mathrm{~mm}, 25 \mathrm{~mm}$ and $50 \mathrm{~mm}$. A 2-cycle sine wave with a center frequency of $5 \mathrm{MHz}$ is used as the excitation. The SHI is achieved by using Pulse Inversion (PI) technique. The data received with and without PI from SARUS are beamformed using Dynamic Receive Focusing (DRF) and SASB by a Beamformation Toolbox. The Full Widths at Half Maximum (FWHM) in both the lateral and axial directions for these four wire targets using different imaging algorithms (DRF, DRF+SHI, SASB and SASB+SHI) are calculated and shown in the paper. The Full Width at One Tenth Maximum (FWOTM) is also investigated. By combining SASB and SHI, the lateral resolution is improved by $66 \%, 35 \%$ and $46 \%$ for FWHM, and $52 \%, 20 \%$ and $29 \%$ for FWOTM, compared to DRF, DRF+SHI and SASB, respectively. The axial resolution is improved $24 \%$ on average by SHI.
\end{abstract}

\section{INTRODUCTION}

Non-linear ultrasound may be combined with different imaging methods to further improve the resolution. The conventional harmonic imaging uses a fixed transmit focus and dynamic receive focus (DRF). Synthetic aperture imaging is a well-know ultrasound imaging method, where dynamic focusing can be achieved in both transmit and receive. Synthetic aperture harmonic imaging has been introduced by Bae et al [1] in 2008. In conventional synthetic aperture imaging, a single element is used for emissions to simulate a spherical wave. The major drawback is that the transmitting energy for a single element is too low, so that the harmonic signals are very weak. Although there can be a multi-element emission used to enhance the transmitting energy and simulate a virtual focal point behind or in front of the transducer, it still requires a lot of memory for saving the data for each low resolution image, when using the conventional synthetic aperture imaging.

Synthetic aperture sequential beamforming (SASB) is a novel technique, also called dual-stage beamforming, which has been researched and developed by Kortbek et al [2], [3] in 2007. The advantage of SASB is that the lateral resolution is improved independently of image depth compared to the conventional ultrasound imaging using DRF. SASB can be

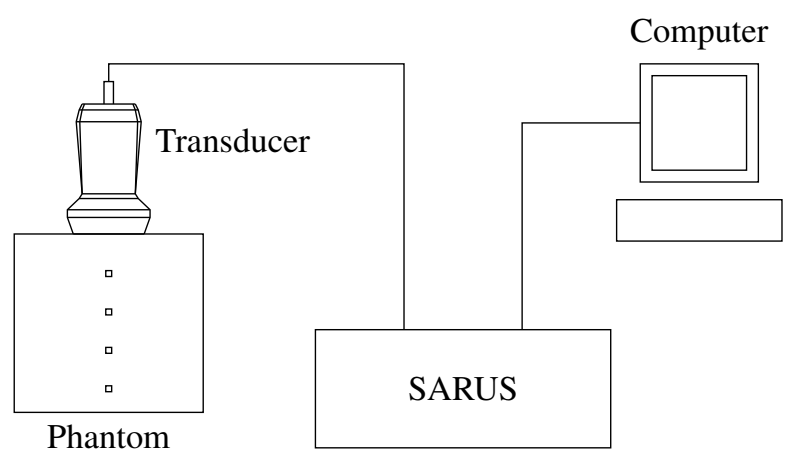

Fig. 1: Measurement setup - linear array transducer, wire phantom under water, SARUS (synthetic aperture real-time ultrasound system).

thought of as a two-stage procedure. The first stage is to obtain a set of conventional B-mode image lines with a fixed transmit and receive focal point. Then the image is created by synthetic aperture imaging using the B-mode image lines in the second stage. In this way, not only is dynamic focusing in both transmit and receive achieved, but the memory requirement is also reduced, since each low resolution image is only stored in one B-mode image line. In the first stage of SASB, a number of elements are used for a fixed focus transmission. Thus, a high penetration depth and SNR for harmonic imaging can be attained.

The purpose of this study is to investigate harmonic imaging using the dual-stage synthetic aperture sequential beamforming. The measured results using different imaging methods are illustrated and compared in the following sections. A discussion based on the measured results and a final conclusion are given at the end of the paper.

\section{METHOD}

The measurement setup is illustrated in Fig. 1. The investigation is made by an experimental scanner - Synthetic Aperture Real-time Ultrasound System (SARUS) [4]. A linear array transducer (BK8804, from BK Medical Aps) is used to transmit and receive data from a wire phantom in water. Four wires are used as point targets at the image depths of $22.5 \mathrm{~mm}, 47.5 \mathrm{~mm}, 72.5 \mathrm{~mm}$ and $97.5 \mathrm{~mm}$, respectively. Three different experiments are made using three different transmit 
foci, which are $10 \mathrm{~mm}, 25 \mathrm{~mm}$ and $50 \mathrm{~mm}$ from the transducer surface. A two-cycle sine wave with a center frequency of $5 \mathrm{MHz}$ is used as the excitation.

Four imaging methods are investigated: dynamic receive focused imaging (DRFI), dynamic receive focused second harmonic imaging (DRFSHI), synthetic aperture sequential beamformed imaging (SASBI), and synthetic aperture sequential beamformed second harmonic imaging (SASBSHI). The second harmonic imaging is achieved using a pulse inversion technique [5], [6]. This is made by using two excitations with a $180^{\circ}$ phase difference, sending them in turn for each emission and summing the two received RF lines. The odd harmonic components (1st(fundamental), 3th, 5th, etc.) are canceled out during this process. Then a matched filter is applied to remove the higher even harmonic components (4th, 6th, 8th, etc.) and leave the second harmonic component only. The received RF lines obtained from SARUS after pulse inversion are postprocessed by a Beamformation Toolbox [7], [8] to achieve the DRF and SASB.
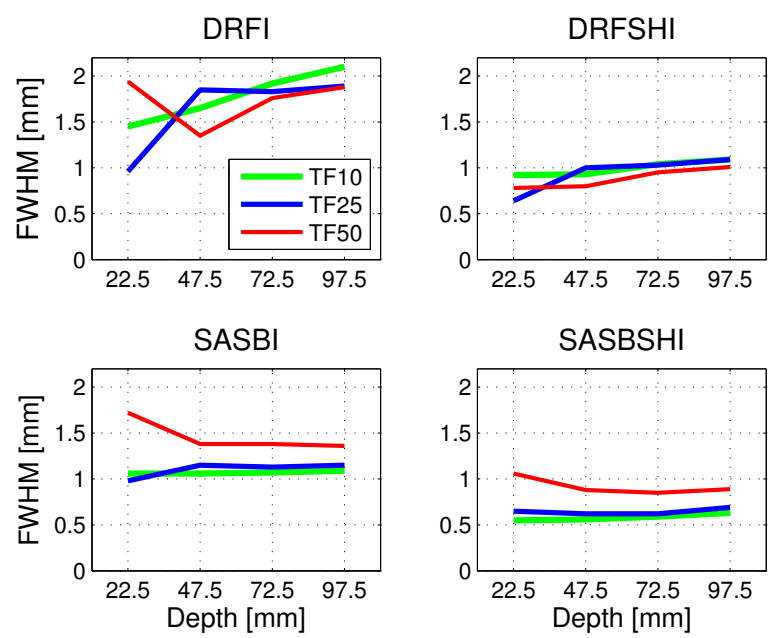

Fig. 3: Lateral FWHM for 4 points along depth - TF10, TF25 and TF50 denote transmit foci are at $10 \mathrm{~mm}, 25 \mathrm{~mm}$ and $50 \mathrm{~mm}$.
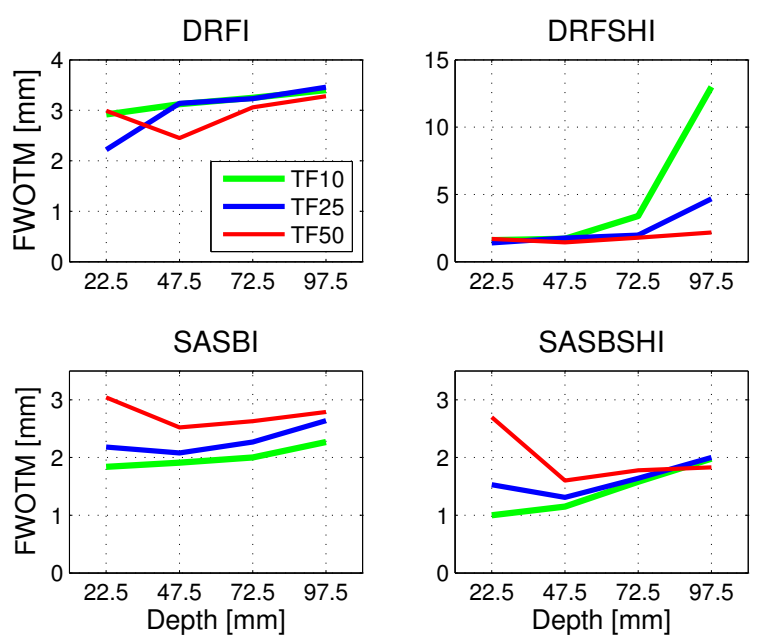

Fig. 4: Lateral FWOTM for 4 points along depth - TF10, TF25 and TF50 denote transmit foci are at $10 \mathrm{~mm}, 25 \mathrm{~mm}$ and $50 \mathrm{~mm}$.

\section{RESULTS}

The images for the wire phantom using the different methods are shown in Fig. 2. The FWHM (full width at half maximum (at $-6 \mathrm{~dB})$ ) and FWOTM (full width at one tenth maximum (at $-20 \mathrm{~dB})$ ) along the lateral direction are calculated for each point with different methods and transmit foci, and plotted in Figs. 3 and 4. The comparison among these 4 imaging methods should be made using the best possible setup for each method. Three measurements with different transmit foci were made for each method. The FWHM and FWOTM for these 4 measured points and the average resolution as a function of depth are evaluated for different transmit foci in each method. After the evaluation, the "best" image with an optimized transmit foci is used to compare with the other imaging methods. The optimization is performed by comparing the FWHM and FWOTM for each point. The shortest FWHM and FWOTM will get 1 point, and no point for others, so that there will be eight ( 4 for FWHM and 4 for FWOTM) comparisons among 3 transmit foci. The total score will be divided by its average FWHM in millimeter. The final scores are shown in Table I.

TABLE I: Scores of the image quality using different transmit foci, where A - FWHM is the average FWHM for these 4 points.

\begin{tabular}{c||ccc}
\hline \hline \multicolumn{1}{c||}{ TF } & $10 \mathrm{~mm}$ & $25 \mathrm{~mm}$ & $50 \mathrm{~mm}$ \\
\hline Subscore & 0 & DRFI \\
A - FWHM & 1.78 & 1.63 & 1.73 \\
Final score & 0 & 1.23 & 3.47 \\
\hline \hline \multicolumn{1}{c||}{} & \multicolumn{3}{c}{ DRFSHI } \\
\hline Subscore & 0 & 2 \\
A - FWHM & 0.99 & 0.94 & 0.89 \\
Final score & 0 & 2.13 & 6.74 \\
\hline \hline & \multicolumn{3}{|}{ SASBI } \\
\hline Subscore & 7 & 1 \\
A - FWHM & 1.07 & 1.10 & 1.46 \\
Final score & 6.54 & 0.91 & 0 \\
\hline \hline & \multicolumn{3}{|c}{ SASBSHI } \\
\hline Subscore & 7 & 0 \\
A - FWHM & 0.58 & 0.64 & 0.92 \\
Final score & 12.1 & 0 & 1.09 \\
\hline
\end{tabular}

Hereby, the final comparison between the different imaging methods is made among DRFI (TF: $50 \mathrm{~mm}$ ), DRFSHI (TF: $50 \mathrm{~mm}$ ), SASBI (TF: $10 \mathrm{~mm})$ and SASBSHI (TF: $10 \mathrm{~mm})$ as show in Fig. 5. By calculating the average FWHM and FWOTM for those four points, the lateral resolution of SASBSHI is improved by $66 \%, 35 \%$ and $46 \%$ for FWHM, and $52 \%, 20 \%$ and $29 \%$ for FWOTM compared to DRFI, DRFSHI and SASBI, respectively.

To show the axial resolution, the center image lines, which go through the point target, are plotted for each method for the second wire (P2) as shown in Fig. 6. The envelopes of the pulses using these four methods DRFI, DRFSHI, SASBI and SASBSHI are plotted in Fig. 7a. The received pulse length becomes shorter for harmonic imaging. However, SASBI does not reduce the length of the pulse compared to DRFI. The results for the FWHM in the axial direction using different imaging methods can be found in Fig. 7b. The axial FWHM of SASBSHI is improved by $27 \%, 5 \%$ and $25 \%$ compared to DRFI, DRFSHI and SASBI, respectively. 

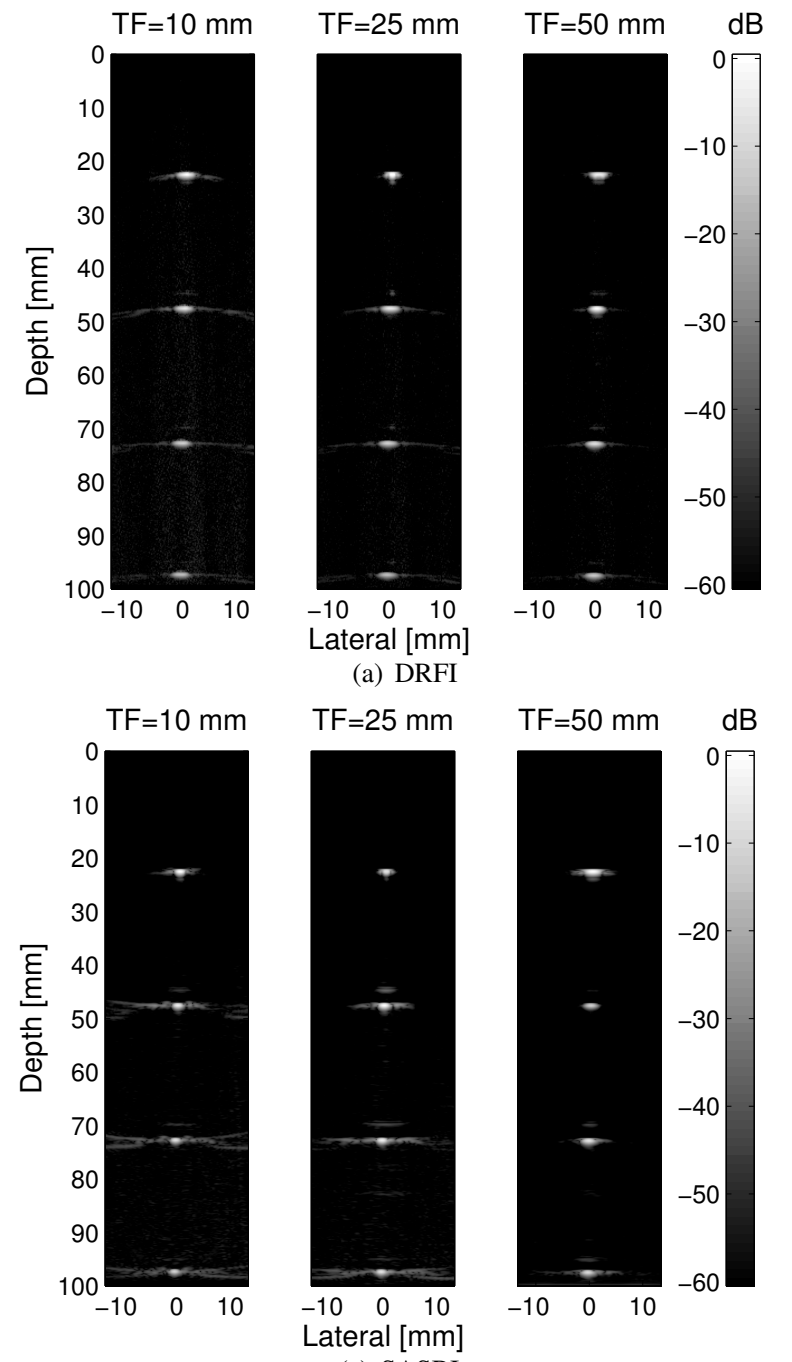

(c) SASBI

Fig. 2: Ultrasound images using the different methods - DRFI: dynamic receive focused imaging, DRFSHI: dynamic receive focused second harmonic imaging, SASBI: synthetic aperture sequential beamformed imaging, SASBSHI: synthetic aperture sequential beamformed second harmonic imaging. TF: transmit focus.

\section{DISCUSSION}

Fig. 5a shows that the SASBSHI gives a minimum FWHM in terms of the image resolution and it maintains the same values along the depth. However, it can be found that the FWOTM using SASBSHI is increased as a function of depth as shown in Fig. 5b. This is because that the harmonic signals are weak at the depth far from the transducer, since the transmit foci is $10 \mathrm{~mm}$ for the SASB. The noise will have much stronger impact on the harmonic signals at $-20 \mathrm{~dB}$ than that at $-6 \mathrm{~dB}$. The previous study of SASB by Kortbek [2] showed that the transmit foci was preferred to be close to the transducer surface to get a better resolution from the SASB. The combination of SASB and SHI is supposed to meet the compromise that the harmonic signals are not reduced too much in the deep depth, meanwhile the transmit foci is still close enough to the transducer to ensure SASB functioning. This can be optimized by doing parameter studies in the future work by trying more different transmit focal distances at the first stage of SASB. The resolution can be investigated by calculating the full width
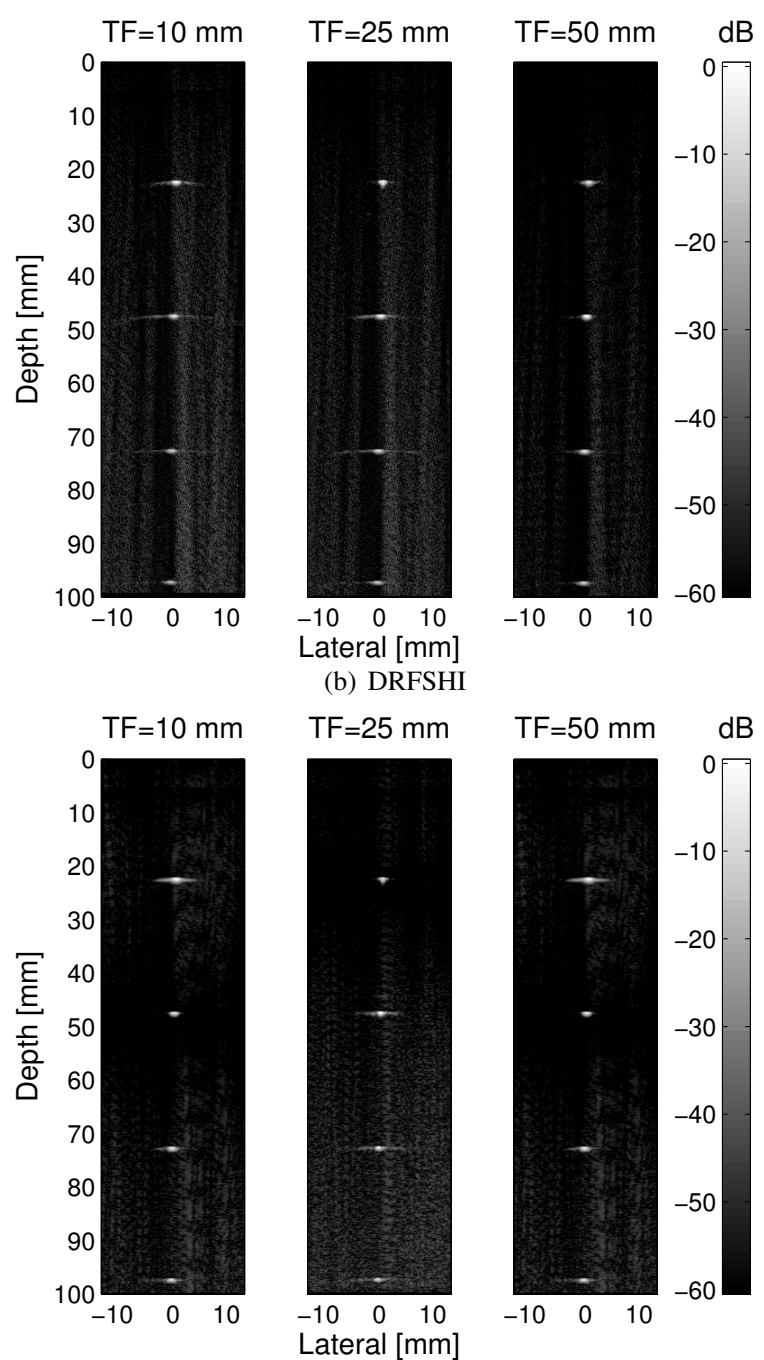

(d) SASBSHI 
(a) Envelops for P2

(a) Lateral FWHM (-6 dB)

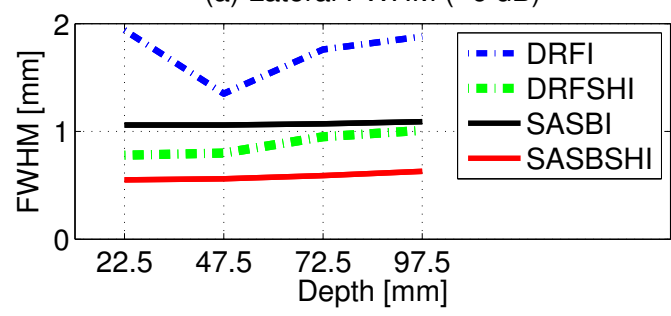

(b) Lateral FWOTM $(-20 \mathrm{~dB})$

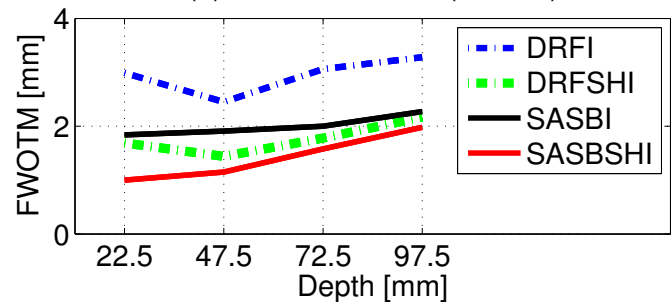

Fig. 5: Lateral full width at $-6 \mathrm{~dB}$ (FWHM - top figure) and -20 $\mathrm{dB}$ (FWOTM - bottom figure) - The transmit foci for the DRFI and DRFSHI is $50 \mathrm{~mm}$ and for the SASBI and SASBSHI it is $10 \mathrm{~mm}$. The 4 wires are at depths of $22.5 \mathrm{~mm}, 47.5 \mathrm{~mm}, 72.5 \mathrm{~mm}$ and $97.5 \mathrm{~mm}$ (a) DRFI for P2 in axial

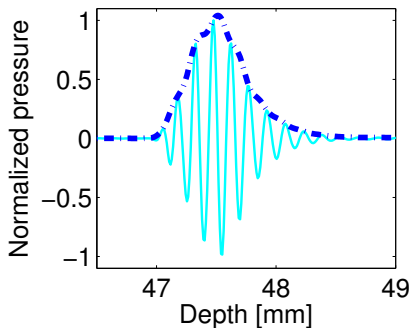

(c) SASBI for P2 in axial

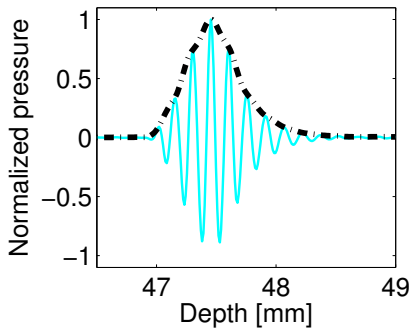

(b) DRFSHI for P2 in axial

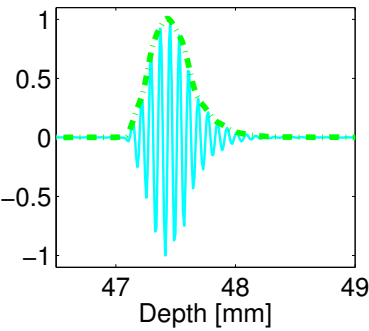

(d) SASBSHI for P2 in axial

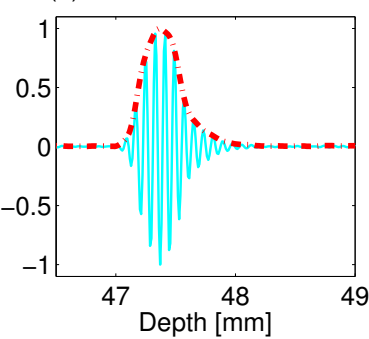

Fig. 6: Pulses and envelops in the axial direction using different imaging methods - These show the center image lines for DRFI, DRFSHI, SASBI, SASBSHI and their envelopes. The plots show the second point target (P2) along the scanning depth and is around $47.5 \mathrm{~mm}$ from the transducer surface. The transmit foci is $50 \mathrm{~mm}$ for DRFI and DRFSHI, and it is $10 \mathrm{~mm}$ for SASBI and SASBSHI.

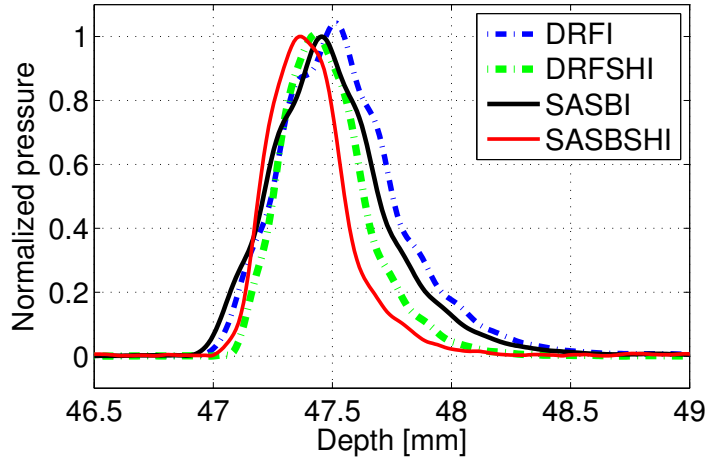

(b) Axial FWHM (-6 dB)

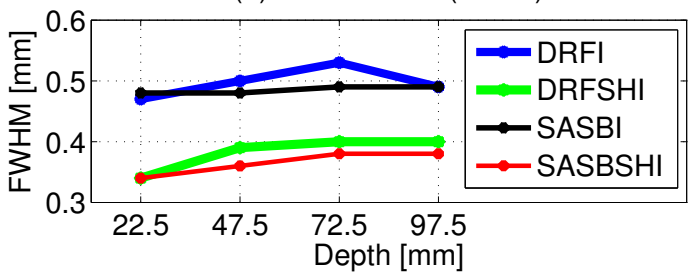

Fig. 7: Envelops and axial FWHM - The envelopes for DRFI, DRFSHI, SASBI and SASBSHI are plotted together in the top figure. The values of the axial FWHM for each method are shown in the bottom figure.

\section{ACKNOWLEDGMENT}

This work was supported by grant 08-032480 from the Danish Agency for Science, Technology and Innovation, and by BK Medical Aps, Denmark.

\section{REFERENCES}

[1] M. Bae, H. Lee, S. B. Park, R. Yoon, M. H. Jeong, D. G. Kim, M. Jeong, and Y. Kim. A new ultrasonic synthetic aperture tissue harmonic imaging system. In Proc. IEEE Ultrason. Symp., pages 1258-1261, 2008.

[2] J. Kortbek. Synthetic aperture sequential beamforming and other beamforming techniques in ultrasound imaging. PhD thesis, Technical University of Denmark, Lyngby, Denmark, 2007.

[3] J. Kortbek, J. A. Jensen, and K. L. Gammelmark. Synthetic aperture sequential beamforming. In Proc. IEEE Ultrason. Symp., pages 966-969, 2008.

[4] J. A. Jensen, H. Holten-Lund, R. T. Nielson, B. G. Tomov, M. B. Stuart, S. I. Nikolov, M. Hansen, and U. D. Larsen. Performance of SARUS: a synthetic aperture real-time ultrasound system. In Proc. IEEE Ultrason. Symp., pages 305-309, 2010.

[5] C. S. Chapman and J. C. Lazenby. Ultrasound imaging system employing phase inversion subtraction to enhance the image. US Patent, 5632277, 1997.

[6] D. H. Simpson, C. T. Chin, and P. N. Burns. Pulse inversion Doppler: a new method for detecting nonlinear echoes from microbubble contrast agents. IEEE Trans. Ultrason., Ferroelec., Freq. Contr., 46(2):372-382, 1999.

[7] J. Kortbek, S. I. Nikolov, and J. A. Jensen. Effective and versatile software beamformation toolbox. In Proc. SPIE - Medical Imaging - Ultrasonic Imaging and Signal Processsing, pages 651319,1-10, 2007.

[8] S. Gustavsson and J. Kortbek. User guide for the beamformation toolbox II, release 9.0. Technical report, Technical University of Denmark, Lyngby, Denmark, 2009. 